Research Article

\title{
The Prebiotic-Like Effects of Coprinus comatus Polysaccharides on Gut Microbiota in Normal Mice and Those with Acute Alcoholic Liver Injury: A Comparative Study
}

\author{
Weidong Li, ${ }^{1}$ Yongxia Wang, ${ }^{1}$ Min Sun, ${ }^{1}$ Yuting Liang, ${ }^{1}$ Xiaoqing Cai, ${ }^{1}$ Dongmei Qi, ${ }^{2}$ \\ Yongqing Zhang $\left(\mathbb{D},{ }^{1}\right.$ and Chunchao Han $\left(\mathbb{B}{ }^{1}\right.$ \\ ${ }^{1}$ School of Pharmacy, Shandong University of Traditional Chinese Medicine, Jinan 250355, China \\ ${ }^{2}$ Experimental Center, Shandong University of Traditional Chinese Medicine, Jinan 250355, China \\ Correspondence should be addressed to Yongqing Zhang; zyq622003@126.com and Chunchao Han; chunchaoh@126.com
}

Received 15 June 2020; Revised 11 August 2020; Accepted 7 October 2020; Published 29 November 2020

Academic Editor: Gabriel A. Agbor

Copyright (c) 2020 Weidong Li et al. This is an open access article distributed under the Creative Commons Attribution License, which permits unrestricted use, distribution, and reproduction in any medium, provided the original work is properly cited.

\begin{abstract}
This study aims to investigate the prebiotic-like effects of Coprinus comatus polysaccharides (CCP) on gut microbiota. Mice were divided into four groups: normal group (NG), alcohol group (AG), polysaccharides group (PG), and alcohol + polysaccharides group (APG). The gut microbiota structure of feces was analyzed by determining the V3-V4 region sequence in 16S rDNA. The results showed CCP could increase the diversity of gut microbiota. Compared with NG, PG had a significantly higher relative abundance of Firmicutes and Lactobacillaceae and a lower abundance of Rikenellaceae. These changes in gut microbiota result in positive effects on gut due to a series of prebiotic-like effects of CCP. At the same time, CCP could improve some adverse changes in gut microbiota caused by acute alcohol intake, such as the increased proportion of Firmicutes, Bacteroidetes, Muribaculaceae, and Lachnospiraceae and the decreased proportion of Rikenellaceae. In conclusion, the CCP has certain prebiotic effects not only on normal mice but also on mice with acute alcoholic liver injury.
\end{abstract}

\section{Introduction}

The consumption of alcohol has been rising with the development of the economy, which leads to a massive increase in the number of alcoholic liver injury cases. Patients with alcoholic liver injury frequently develop into liver disease such as liver fibrosis and cirrhosis, which could increase the risk of cancer [1]. The liver is also the organ most closely associated with the gut, and various liver diseases have been proved to be related to the altered gut microbiota [2].

Gut microbiota plays an important role in disease such as obesity, inflammatory bowel diseases (IBD), and liver cancer [3]. As an endocrine system, the gut microbiota communicates with other organs through a bidirectional communication system [4]. These communications are crucial to safeguarding the function of body, such as metabolic reactions, immune regulation, and pathogen elimination [5]. Hence, researchers paid more attention to designing and developing some innovative interventions to modulate gut microbiota, which could promote beneficial microbial-host interactions. Although the age, sex, and genes are important factors which are difficult to be changed, external environment could directly influence the gut microbiota such as prebiotics.

Research in the area of prebiotic interventions has increased exponentially in recent decades. As defined by the International Scientific Association, prebiotics are healthful substrates selectively used by host microorganisms [6]. The Coprinus comatus (C. comatus, Agaricaceae, and Agaricomycetes) is a new type of edible fungus in the north of China, which is used for food and medicine. C. comatus has some high nutritional ingredients, including polysaccharides, polyphenols, fatty acids, tocopherols, and organic acids. Especially, C. comatus polysaccharide (CCP) has been proven to contain activities, including antioxidation, hypoglycemic effect, and protection of the liver [7-9]. 
However, there are few studies on the probiotic-like effects of CCP on gut microbiota. Therefore, our experiment aims to compare the prebiotic-like effects of CCP in normal mice and those with acute alcoholic liver injury to observe the effect of CCP on gut microbiota.

\section{Materials and Methods}

2.1. Extraction of Polysaccharides from C. comatus. The fruiting body of $C$. comatus was provided by Jiangsu Chenxi Biotechnology Co., Ltd. (Jiangsu China). Polysaccharides fractions were extracted stepwise following the method of $\mathrm{Li}$ et al. [10] with some modifications. Dried in the oven $\left(45^{\circ} \mathrm{C}\right)$, C. comatus was extracted twice with distilled water according to the ratio of $1: 2$ at $90^{\circ} \mathrm{C}$ for $3 \mathrm{~h}$. After cooling to room temperature, the extract was mixed and centrifuged to obtain supernatants. The supernatants were concentrated to $1 / 4$ of the original volume in the rotary evaporator. Then, the concentrated solution was mixed with absolute ethyl alcohol at $4^{\circ} \mathrm{C}$ overnight. Subsequently, through centrifugation at $4000 \mathrm{rpm}$ for $15 \mathrm{~min}$, the precipitate was obtained and washed with absolute ethyl alcohol and acetone consecutively. Redissolved in distilled water, the washed precipitates were mixed with a quarter volume of Sevag Reagent (Tianjin Fuyu Fine Chemical Co., Ltdj) to remove the proteins. The aqueous phase was then mixed with absolute ethyl alcohol at $4^{\circ} \mathrm{C}$ overnight to obtain the precipitation through centrifugation. After washing with acetone and petroleum ether, the precipitation was redissolved in distilled water and lyophilized to obtain the purified C. comatus polysaccharides.

2.2. Animal. Kunming (KM) mice were purchased from the Beijing Vital River Laboratory Animal Technology Co., Ltd. (Beijing, China). All of the mice were male, 4-5 weeks old, and weighing $20 \pm 2 \mathrm{~g}$. Standards for Animal Experimentation of Shandong University of Traditional Chinese Medicine were used in all experiments, which were in conformity to the rules of the Animal Ethics Committee of the institution (Shandong, China). The mice adapted the environment for seven days adaptively before the formal experiment. The states of the living environment are listed as follows: the living environment was specific pathogen free (SPF) grade. We maintained a schedule under a condition of $12 / 12 \mathrm{~h}$ light/dark. The mice room temperature was maintained at $18-20^{\circ} \mathrm{C}$ with a relative humidity of $40 \%$ to $60 \%$. Animal feeding was according to the standard protocol. The room was ventilated on time, and all mice received humane care.

2.3. Experimental Design. After adapting the environment for 7 days, the mice were divided into four groups: NG (normal group), AG (alcohol group), PG (polysaccharides group), and APG (alcohol + polysaccharides group) ( $n=10$ per group). The PG and APG were orally administered by CCP (200 mg per kg bw) for 30 days. The NG and AG were given the same volume of distilled water for 30 days intragastrically. On the thirtieth day, the mice in each group were fasted for 16 hours overnight before modeling. After fasting, the AG and APG were given 50\% ethanol $(10 \mathrm{ml}$ per $\mathrm{kg}$ bw) intragastrically, and the NG and PG were given the same volume of distilled water. After 24 hours of acute alcohol modeling, the fecal samples of mice were collected by the sterile EP tube. All fecal samples were stored at $-80^{\circ} \mathrm{C}$. Blood samples were collected and centrifuged at $3000 \mathrm{rpm}$ for $10 \mathrm{~min}$ to separate serum for further analysis.

2.4. Determination of Biochemical Indexes. Serum alanine aminotransferase (ALT) and aspartate aminotransferase (AST) were analyzed using commercial kits according to the manufacturer's protocols (Rsbio, Shanghai, China).

2.5. DNA Extraction and $16 S$ rDNA Gene Sequencing. HiPure Stool DNA Kits (Magen, Guangzhou, China) were used for extracting microbial DNA according to the manufacturer's protocols. The V3-V4 regions of the 16S rRNA were amplified with primers (341F:CCTACGGG NGGCWGCAG and 806R:GGACTACHVGG-GTATCTAAT) [11]. Subsequently, purified amplicons were pooled in equimolar amounts, and paired-end was sequenced on an Illumina platform according to standard protocols described by Gene Denovo Biotechnology Co., Ltd. (Guangzhou, China). The raw reads were further filtered through FASTP and merged as raw tags using FLSAH $[12,13]$. Chimeric sequences were identified and removed using UCHIME [14]. UPARSE pipeline was used for assembling the effective tags into operational taxonomic units (OTUs) of $\geq 97 \%$ similarity [15]. The taxonomical assignment of OTUs was performed by the RDP classifier algorithm against the Silva database [16]. The analysis such as species annotation, $\alpha$ diversity analysis, and $\beta$ diversity analysis was carried out successively according to the analysis process of OTUs.

2.6. Statistical Analysis. All data are expressed as the mean \pm SD (standard deviations). Significant differences between two groups were assessed by using the Student's $t$ test. Comparison between multiple groups was analyzed by using the one-way ANOVA test followed by the Student-Newman-Keuls (SNK) test. Data were collected and analyzed using Statistical Product and Service Solutions (SPSS) 26.0 software (SPSS Inc., Chicago, IL, USA). $P<0.05$ was considered statistically significant.

\section{Results and Discussion}

3.1. Biochemical Indicators of Liver Injury. The serum ALT and AST levels, as biochemical indicators of liver injury, were significant increased in alcohol-treated mice $(P<0.05)$ (Figure 1).

The default provided $97 \%$ consistency to cluster the sequence into OTUs (Operational Taxonomic Units) with an average of 1999 per sample. Alpha diversity was used for reflecting richness and diversity of gut microbiota through ACE, Chao1, Shannon diversity index, and Simpson diversity index. The richness of gut microbiota (Figures 2(a) and 2(b)) in PG and APG was increased compared with NG, 


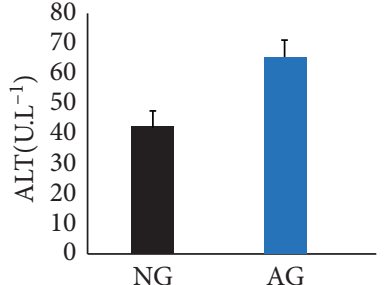

(a)

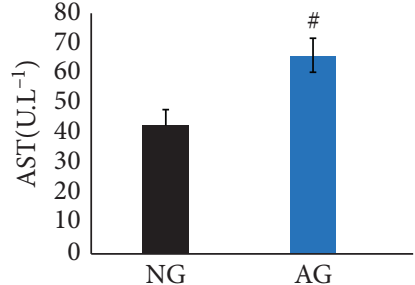

(b)

Figure 1: The effects of CCP on serum ALT and AST in mice. Values are presented as mean \pm SD for 10 mice in each group. ${ }^{\#} P<0.05$ as compared with the NG.

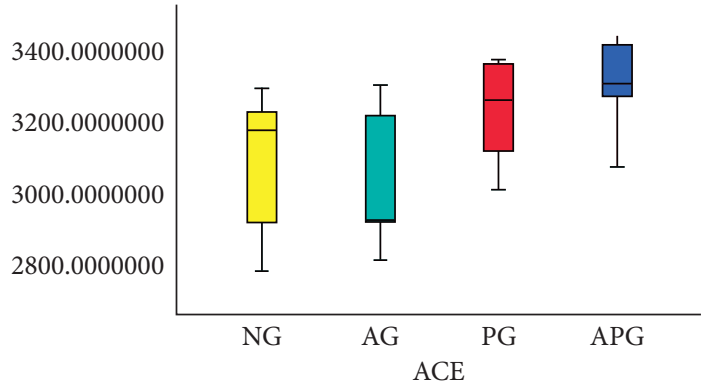

(a)

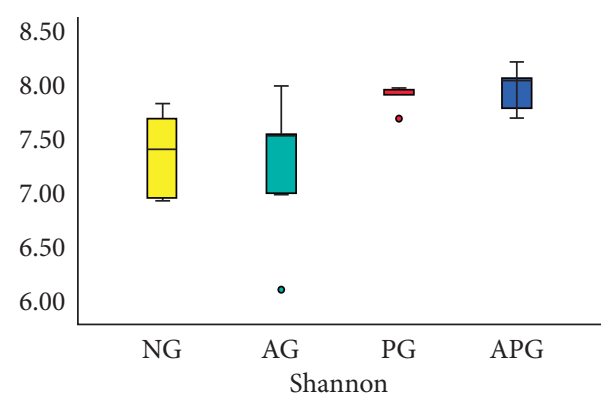

(c)

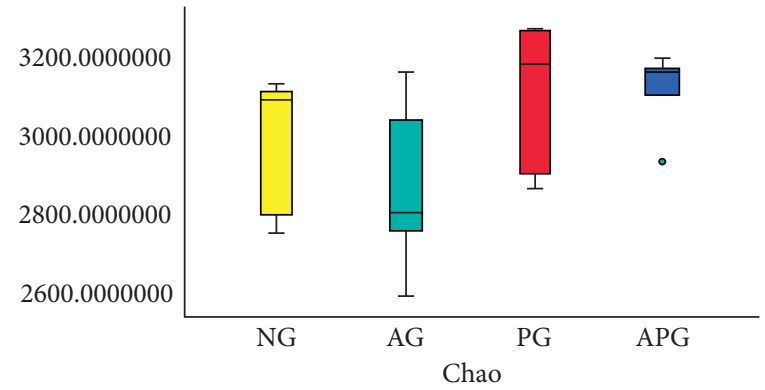

(b)

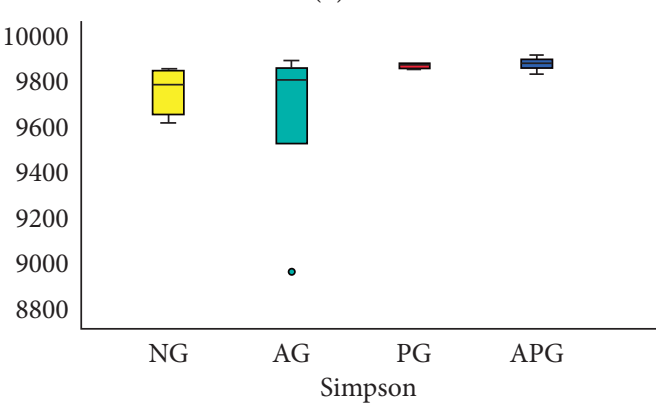

(d)

FIGURe 2: Differences in gut microbial diversity and richness of NG, AG, PG, and APG. (a) ACE; (b) Chao; (c) Shannon diversity; (d) Simpson diversity. ${ }^{\#} P<0.05$ as compared with the NG.

but there was no significant difference. However, the data of Shannon diversity (Figures 2(c) and 2(d)) indicated that the diversity of gut microbiota in APG and PG was significantly higher than NG $(P<0.05)$. Meanwhile, AG was not different from NG in the richness and diversity of gut microbiota according to Figure 2.

3.2. Composition of Gut Bacteria. At the phylum level (Figure 3), the two principal phyla of gut microbiota were Bacteroidetes (59.94\%) and Firmicutes (37.73\%) in the NG. Compared with NG, the proportion of Bacteroidetes was reduced by $15.77 \%$ in AG, $10.18 \%$ in APG, and $4.97 \%$ in PG. Compared with NG, the ratio of Firmicutes was increased by $6.58 \%$ in APG and $4.18 \%$ in PG, and decreased by $1.58 \%$ in AG. Furthermore, the relative proportion of Verrucomicrobia in AG was significantly higher than that of the other three groups $(P<0.05)$.
At the family level (Figure 4), the two principal phyla of gut microbiota were Muribaculaceae (41.11\%) and Lachnospiraceae $(26.51 \%)$ in the NG. There was no significant difference in the proportion of Muribaculaceae between NG and PG. But compared with NG, the proportion of Muribaculaceae in AG and APG was reduced by $17.27 \%$ and $9.07 \%$ $(P<0.05)$. At the same time, the proportion of Lachnospiraceae in AG was decreased by $8.75 \%$ compared with NG $(P<0.05)$. But, there were no significant differences among the other three groups except AG. And, the content of Rikenellaceae was obviously different among AG (5.00\%), APG (4.45\%), NG $(2.50 \%)$, and PG $(2.30 \%)(P<0.05)$. Furthermore, Lactobacillaceae of PG (3.09\%) showed a significant increase compared with NG (1.87\%), AG (0.67\%), and APG (0.48\%). Meanwhile, the proportion of Akkermansiaceae in APG (2.99\%) was 4 times that of NG $(0.74 \%)$ and 6 times of PG $(0.45 \%)$, and alcohol intake induced an abnormal rise of Akkermansiaceae compared with other groups. 


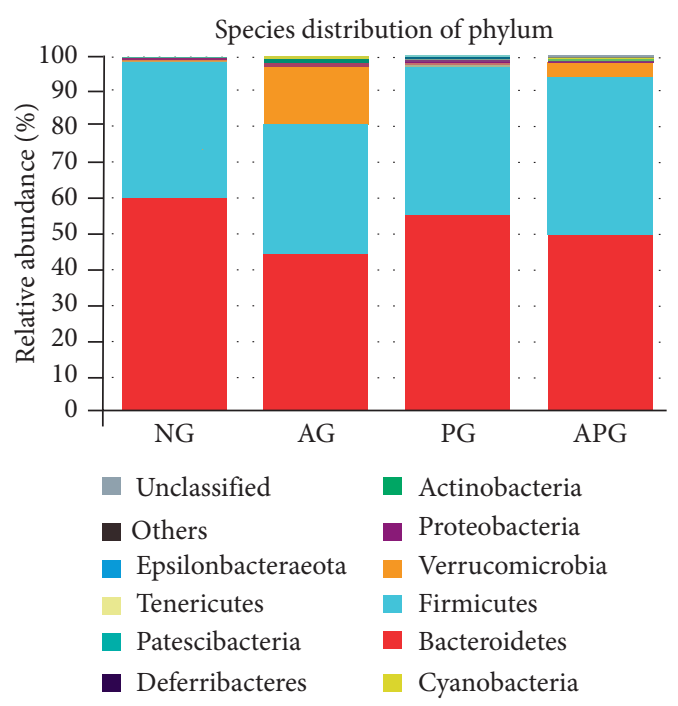

FIgURE 3: The relative abundance of gut microbiota at the phylum level.

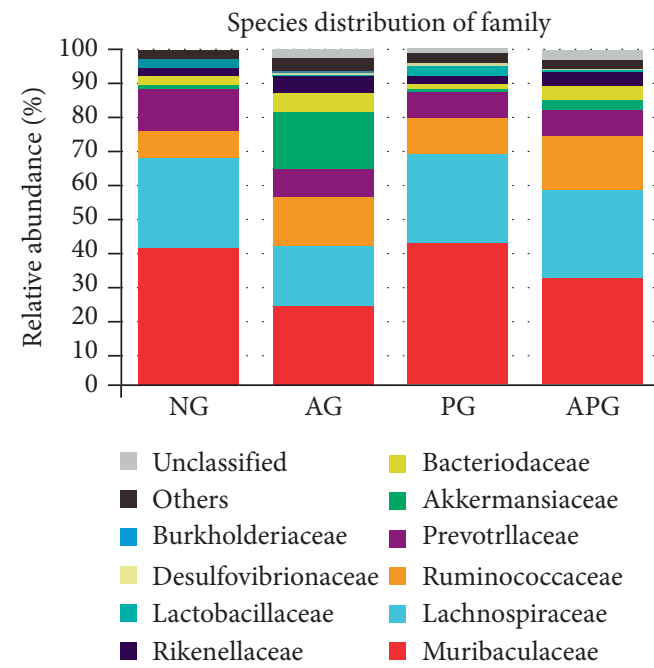

FIgUre 4: The relative abundance of gut microbiota at the family level.

At the genus level (Figure 5), the content of Akkermansia in APG (2.99\%) was 4 times that of NG $(0.74 \%)$ and 6.5 times of PG $(0.46 \%)$. Alcohol intake induced an abnormal rise of Akkermansia compared with other groups $(P<0.01)$. The populations of Lactobacillus in PG (3.08\%) were significantly increased compared with NG (1.87\%). But, alcohol intake could decrease the populations of Lactobacillus in AG $(0.67 \%)$ significantly, which could not be improved in APG $(0.48 \%)$ by CCP.

\section{Discussion}

The experiment aims to compare the prebiotic-like effects of $\mathrm{CCP}$ on gut microbiota in normal mice and those with acute alcoholic liver injury to explore the effect of CCP on gut microbiota. The results showed that the CCP could promote

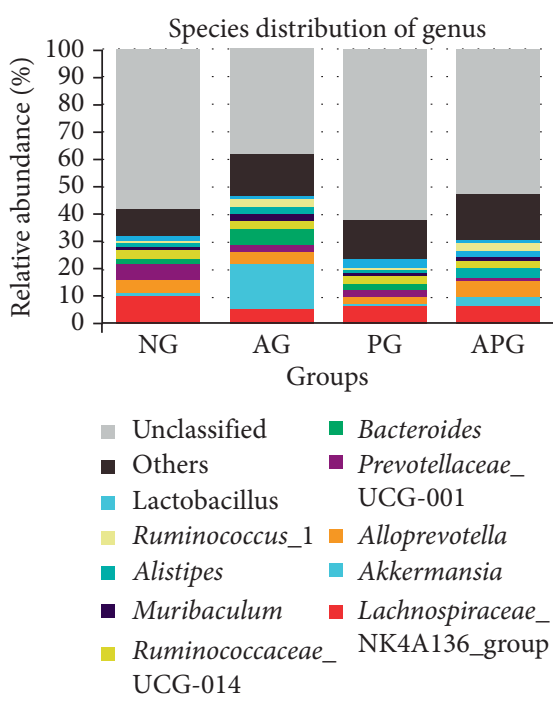

FIgURE 5: The relative abundance of gut microbiota at the genus level.

beneficial gut microbiota to some extent in normal mice and mice with acute alcoholic liver injury. However, the CCP could not adjust the gut microbiota of AG to normal state completely.

ALT and AST in serum are important indexes to evaluate liver injury. The previous studies have proven that patients with severe alcoholic liver injury present an increase in serum levels of ALT and AST in comparison with healthy individuals $[17,18]$. In the present study, ALT and AST levels of AG was increased significantly compared with those of NG $(P<0.05)$. Hence, our results proved the alcohol intake caused hepatotoxicity in the mice and the model of alcoholic liver injury was successful.

The alpha diversity (Chao, ACE, Shannon diversity, and Simpson diversity) of gut microbiota in APG and PG was increased compared with that of NG and AG, but only the significant differences of Shannon diversity was found in the data. We infer that the CCP could not increase the richness of gut microbiota, but it could improve the diversity of gut microbiota to some extent.

Bacteroidetes and Firmicutes were the two most abundant phyla among the all experimental groups. In previous studies, the changes in Bacteroidetes were not certain after chronic alcohol feeding. However, the proportion of Firmicutes was decreased under the same conditions [19-21]. Our results showed CCP could increase Firmicute level under normal feeding and alcohol intaking conditions. It has been reported Firmicute could produce more butyrate which is considered a health-promoting molecule. Meanwhile, CCP could improve the reduction in Bacteroidetes induced by alcohol. Bacteroidetes could produce more propionate to promote hepatic gluconeogenesis which could regulate the hypoglycemia caused by alcohol. Hence, we could preliminarily deduce the CCP could adjust the changes of two main phyla to produce prebiotic-like effects [22-24]. Verrucomicrobia was considered as a probiotic, which showed a negative correlation with hepatic inflammation and oxidative stress $[25,26]$. Especially, Akkermansiaceae, the most 
representative gut microbe in the Verrucomicrobia, could prevent the weight gain caused by a high-fat diet, repair the damaged integrity of the intestinal epithelium barrier, reduce endotoxin levels in the blood, and improve insulin resistance [27, 28]. However, an interesting discovery we observed was that alcohol intake did not decrease the proportion of Verrucomicrobia but rather led to an obvious increase. At the same time, CCP had no significant effects on Verrucomicrobia under normal state. Through the analysis of individual data, we found that the abnormal increase of the Verrucomicrobia level was caused by one mouse in AG. By excluding the abnormal data, we found that CCP could inhibit the reduction of Verrucomicrobia caused by alcohol.

Muribaculacea played an important role in maintaining the gut balance. It could promote the production of the short-chain fatty acid (SCFA) [29], which can adjust the gut immune responses and protect the integrity of intestinal epithelial [30]. In our study, acute alcohol intake decreased Muribaculacea significantly, which could contribute to the destruction of normal intestinal function. However, CCP can significantly mitigate the adverse impact caused by alcohol, although it can not affect the proportion of Muribaculacea in normal state. Lachnospiraceae was another important bacterium for producing SCFA. The level of Lachnospiraceae was decreased in AG compared with NG, which could bring about adverse effects on the gut [31]. However, CCP could also alleviate the downward trend of Lachnospiraceae to ameliorate the adverse effects on the gut [32]. The previous study showed Nur77 knockout mice became obese accompanied with the relative abundances of increased Rikenellaceae [33]. At the same time, the abundance of Rikenellaceae was positively correlated with the hepatic inflammation [34]. In the current study, CCP could regulate Rikenellaceae properly to improve physical condition. Meanwhile, Lactobacillaceae could regulate the gut microbiota, enhance the immunity function, improve the gut function, and reduce the inflammation [35-37]. The proportion of Lactobacillaceae in PG was increased considerably compared with that in NG. Meanwhile, the same changes were observed in Lactobacillus at the genus level. Therefore, we inferred that CCP could promote the Lactobacillaceae level in the normal state.

\section{Conclusions}

In conclusion, the effect of CCP on gut microbiota was studied by comparing the prebiotic-like effects of CCP in normal mice and those with acute alcoholic liver injury. The prebiotic-like effects of CCP not only promote the health of gut under normal conditions but also improve some adverse changes in gut microbiota caused by acute alcohol intake. These functions are achieved by promoting beneficial microbiota and inhibiting harmful microbiota by CCP. Hence, we could further explore the development of CCP as a functional food for daily drinking to maintain a balance of gut microbiota. In addition, due to the complex relationship among dietary nutrition, gut microbiota, and host, uncovering specific effects of CCP on the gut still needs more evidence.

\section{Data Availability}

The data used to support the findings of this study are included within the article.

\section{Conflicts of Interest}

The authors declare that there are no conflicts of interest.

\section{Acknowledgments}

This work was supported by the project of Shandong Province Key Research and Development Program (Grant no. 2017YYSP030), Shandong Provincial Natural Science Foundation, China (No. ZR2017MH086), Major Science and Technology Innovation in Shandong Province (Grant no. 2017CXGC1307), Ji'nan Science and Technology Project (Grant no. 201303055), Key Laboratory of Classical Theory of Traditional Chinese Medicine, Ministry of Education, Shandong Provincial Key Laboratory of Traditional Chinese Medicine for Basic Research, and Traditional Chinese Medicine Resources and Utilization Innovation Team.

\section{References}

[1] V. Purohit and D. A. Brenner, "Mechanisms of alcohol-induced hepatic fibrosis: a summary of the Ron Thurman symposium," Hepatology, vol. 43, no. 4, pp. 872-878, 2006.

[2] H. Til, P. D. Cani, E. A. Mayer et al., "Gut microbiome and liver diseases," Gut, vol. 65, no. 12, pp. 2035-2044, 2016.

[3] R. Villette, KC. Pukar, S. Beliard et al., "Unraveling host-gut microbiota dialogue and its impact on cholesterol levels," Frontiers in Pharmacology, vol. 11, 2020.

[4] P. D. Cani and K. Claude, "How gut microbes talk to organs: the role of endocrine and nervous routes," Molecular Metabolism, vol. 5, pp. 743-752, 2016.

[5] L. Chen, W. C. S. Tai, and W. L. W. Hsiao, "Dietary saponins from four popular herbal tea exert prebiotic-like effects on gut microbiota in C57BL/6 mice," Journal of Functional Foods, vol. 17, pp. 892-902, 2015.

[6] G. R. Gibson, R. Hutkins, M. E. Sanders et al., "Expert consensus document: the International Scientific Association for Probiotics and Prebiotics (ISAPP) consensus statement on the definition and scope of prebiotics," Nature Reviews Gastroenterology \& Hepatology, vol. 14, no. 8, pp. 491-502, 2017.

[7] Z. Huajie, Z. Jianjun, L. Xinchao, Y. Qihang, D. Yuhan, and J. Le, "The antioxidant activities of alkalic-extractable polysaccharides from Coprinus comatus on alcohol-induced liver injury in mice," Scientific Reports, vol. 8, no. 1, Article ID 11695, 2018.

[8] H. Zhao, H. Li, Q. Lai, Q. Yang et al., "Antioxidant and hepatoprotective activities of modified polysaccharides from Coprinus comatus in mice with alcohol-induced liver injury," International Journal of Biological Macromolecules, vol. 127, pp. 476-485, 2019.

[9] H. Lai, S. Ma, H. Guo et al., "Comparative study on the monosaccharide compositions, antioxidant and hypoglycemic activities in vitro of intracellular and extracellular polysaccharides of liquid fermented Coprinus comatus," International Journal of Biological macromolecules, vol. 139, pp. 543-549, 2019. 
[10] B. Li, M. D. Justyna, G. J. Gerwig, L. Dijkhuizen, and J. P. Kamerling, "Structural investigation of water-soluble polysaccharides extracted from the fruit bodies of Coprinus comatus," Carbohydrate Polymers, vol. 91, no. 1, pp. 314-321, 2013.

[11] M. Guo, F. Wu, G. Hao et al., "Bacillus subtilis improves immunity and disease resistance in rabbits," Frontiers in Immunology, vol. 8, 2017.

[12] S. Chen, Z. Yanqing, C. Yaru, and G. Jia, "Fastp: an ultra-fast all-in-one FASTQ preprocessor," Bioinformatics (Oxford, England), vol. 34, no. 17, pp. i884-i890, 2018.

[13] T. Magoc and S. L. Salzberg, "FLASH: fast length adjustment of short reads to improve genome assemblies," Bioinformatics, vol. 27, no. 21, pp. 2957-2963, 2011.

[14] R. C. Edgar, B. J. Haas, J. C. Clemente, C. Quince, and R. Knight, "UCHIME improves sensitivity and speed of chimera detection," Bioinformatics (Oxford, England), vol. 27, no. 16, pp. 2194-2200, 2011.

[15] R. C. Edgar, "UPARSE: highly accurate OTU sequences from microbial amplicon reads," Nature Methods, vol. 10, no. 10, pp. 996-998, 2013.

[16] Q. Wang, M. G. George, M. T. James, and R. C. James, "Naive Bayesian classifier for rapid assignment of rRNA sequences into the new bacterial taxonomy," Applied and Environmental Microbiology, vol. 73, no. 16, pp. 5261-5267, 2007.

[17] Z. Lou, J. Wang, Y. Chen et al., "Linderae radix ethanol extract attenuates alcoholic liver injury via attenuating inflammation and regulating gut microbiota in rats," Brazilian Journal of Medical and Biological Research, vol. 52, no. 6, Article ID e7628, 2019.

[18] L. Zhao, Y. Jiang, Y. Ni et al., "Protective effects of Lactobacillus plantarum C88 on chronic ethanol-induced liver injury in mice," Journal of Functional Foods, vol. 35, pp. 97-104, 2017.

[19] E. Smirnova, P. Puri, M. D. Muthiah et al., "Fecal microbiome distinguishes alcohol consumption from alcoholic hepatitis but does not discriminate disease severity," Hepatology, vol. 72, no. 1, pp. 271-286, 2020.

[20] S. Bluemel, L. Wang, C. Kuelbs et al., "Intestinal and hepatic microbiota changes associated with chronic ethanol administration in mice," Gut Microbes, vol. 11, no. 3, pp. 265-275, 2019.

[21] J. P. Jacobs, T. S. Dong, V. Agopian et al., "Microbiome and bile acid profiles in duodenal aspirates from patients with liver cirrhosis: the microbiome, microbial markers and liver disease study," Hepatology Research, vol. 48, no. 13, pp. 1108-1117, 2018.

[22] L. Crovesy, M. Daniele, L. R. Eliane et al., "Profile of the gut microbiota of adults with obesity: a systematic review," European Journal of Clinical Nutrition, vol. 74, no. 9, pp. 12511262, 2020.

[23] P. J. Turnbaugh, F. Bäckhed, L. Fulton, and J. I. Gordon, "Dietinduced obesity is linked to marked but reversible alterations in the mouse distal gut microbiome," Cell Host \& Microbe, vol. 3, no. 4, pp. 213-223, 2008.

[24] E. F. Murphy, P. D. Cotter, S. Healy et al., "Composition and energy harvesting capacity of the gut microbiota: relationship to diet, obesity and time in mouse models," Gut, vol. 59, no. 12, pp. 1635-1642, 2010.

[25] L. J. Myhill, S. Stolzenbach, H. Mejer et al., "Fermentable dietary fiber promotes helminth infection and exacerbates host inflammatory responses," Journal of Immunology, vol. 204, no. 11, pp. 3042-3055, 2020.
[26] T. Xia, B. Zhang, S. Li, B. Fang et al., "Vinegar extract ameliorates alcohol-induced liver damage associated with the modulation of gut microbiota in mice," Food \& Function, vol. 11, no. 4, pp. 2898-2909, 2020.

[27] Z. Ou, L. Deng, Z. Lu et al., "Protective effects of Akkermansia muciniphila on cognitive deficits and amyloid pathology in a mouse model of Alzheimer's disease," Nutrition \& Diabetes, vol. 10, no. 1, 2020.

[28] S. Fujisaka, S. U. Isao, N. Allah et al., "Bofutsushosan improves gut barrier function with a bloom of Akkermansia muciniphila and improves glucose metabolism in mice with diet-induced obesity," Scientific Reports, vol. 10, no. 1, pp. 1-14, 2020.

[29] J. Meng, B. Santanu, Z. Li et al., "Opioids impair intestinal epithelial repair in HIV-infected humanized mice," Frontiers in Immunology, vol. 10, 2020.

[30] N. T. Baxter, W. S. Alexander, V. Arvind, S. K. Kwi, W. Clive, and M. S. Thomas, "Dynamics of human gut microbiota and short-chain fatty acids in response to dietary interventions with three fermentable fibers," MBio Journal, vol. 10, no. 1, Article ID e02, 2019.

[31] W. Huo, Z. Feng, S. Hu, L. Cui et al., "Effects of polysaccharides from wild morels on immune response and gut microbiota composition in non-treated and cyclophosphamide-treated mice," Food \& Function, vol. 11, no. 5, pp. 4291-4303, 2020.

[32] X. Hu, L. Dong, X. Zhang et al., "Bound phenolics ensure the antihyperglycemic effect of rice bran dietary fiber in $\mathrm{db} / \mathrm{db}$ mice via activating the insulin signaling pathway in skeletal muscle and altering gut microbiota," Journal of Agricultural and Food Chemistry, vol. 68, no. 15, pp. 4387-4398, 2020.

[33] Q. Lv, A. Yang, W. Shi et al., "Calcipotriol and iBRD9 reduce obesity in Nur77 knockout mice by regulating the gut microbiota, improving intestinal mucosal barrier function," International Journal of Obesity, vol. 44, no. 5, pp. 1052-1061, 2020.

[34] Y.-H. Chen, Y.-C. Wang, C.-C. Chiu et al., "Housing condition-associated changes in gut microbiota further affect the host response to diet-induced nonalcoholic fatty liver," The Journal of Nutritional Biochemistry, vol. 79, Article ID 108362, 2020.

[35] I. A. Kirpich, N. V. Solovieva, S. N. Leikhter et al., "Probiotics restore bowel flora and improve liver enzymes in human alcohol-induced liver injury: a pilot study," Alcohol, vol. 42, no. 8, pp. 675-682, 2008.

[36] A. A. Nanji, U. Khettry, and S. M. H. Sadrzadeh, "Lactobacillus feeding reduces endotoxemia and severity of experimental alcoholic liver (disease)," Experimental Biology and Medicine, vol. 205, pp. 243-247, 1994.

[37] Y. Wang, A. Sidhu, Z. Ma, C. McClain, and W. Feng, "Lactobacillus rhamnosus GG culture supernatant ameliorates acute alcohol-induced intestinal permeability and liver injury," American Journal of Physiology-Gastrointestinal and Liver Physiology, vol. 303, no. 1, pp. G32-G41, 2012. 